\title{
Power halo and magnetic shadow in a solar quiet region observed in the $\mathrm{H} \alpha$ line
}

\author{
I. Kontogiannis ${ }^{1,2}$, G. Tsiropoula ${ }^{1}$, and K. Tziotziou ${ }^{1}$ \\ 1 National Observatory of Athens, Institute for Space Applications and Remote Sensing, Lofos Koufos, 15236 Palea Penteli, Greece \\ e-mail: [jkonto;georgia;kostas]@space.noa.gr \\ 2 Department of Astrophysics, Astronomy and Mechanics, Faculty of Physics, National and Kapodistrian University of Athens, \\ 15784 Zografos, Greece
}

Received 7 July 2009 / Accepted 7 December 2009

\section{ABSTRACT}

\begin{abstract}
Context. We investigate the oscillatory behavior of the quiet solar chromosphere and its discrete components in terms of oscillation properties, i.e. network and internetwork. For this purpose, we use a time series of high resolution filtergrams at five wavelengths along the $\mathrm{H} \alpha$ profile, obtained by the Dutch Open Telescope.

Aims. We aim to gain insight on the distribution of power in different period bands and its variation between network and internetwork. Our spectral resolution provides information on the vertical distribution of power, since the $\mathrm{H} \alpha$ line has both photospheric and chromospheric components. We investigate the effect of $\mathrm{H} \alpha$ mottles on chromospheric oscillations, since they are the most prominent feature of the $\mathrm{H} \alpha$ chromosphere and outline inclined magnetic fields.

Methods. We use wavelet and phase difference analyses of $\mathrm{H} \alpha$ intensities and Doppler signals. Two-dimensional power maps in the 3, 5 and 7 min period bands as well as coherence and phase difference maps were constructed.

Results. At photospheric heights, where the $\mathrm{H} \alpha \pm 0.7 \AA$ wing is formed, the 3 and 5 min power is enhanced around the network, and forms power halos. Higher in the chromosphere these areas are replaced by magnetic shadows, i.e. places of power suppression. Interestingly, the power maps show a filamentary structure in the network which correlates very well with mottles. These areas show positive phase differences at the $3 \mathrm{~min}$ period band. At the $5 \mathrm{~min}$ and $7 \mathrm{~min}$ period bands both positive and negative phase differences are obtained with an increased number of pixels with high coherence, indicating the existence of both upward and downward propagating waves.

Conclusions. We attribute our findings to the interaction between acoustic oscillations and the magnetic fields that constitute the magnetic network. The network flux tubes diverge at chromospheric levels and obtain a significant horizontal component, which is betrayed by the presence of mottles. The variation of power reveals the discrete role of the magnetic field at different heights, which guides or suppresses the oscillations, depending on its inclination. Spectral resolution in $\mathrm{H} \alpha$ provides useful information on the coupling between the acoustic sub-canopy atmosphere and the magnetized chromosphere.
\end{abstract}

Key words. Sun: chromosphere - Sun: oscillations

\section{Introduction}

The quiet chromosphere of the Sun displays a distinct network appearance in filter images taken in most of the chromospheric lines. The bright network forms largely cellular patterns also referred to as "cell boundaries", which outline the supergranules. It consists of mainly vertical magnetic flux tubes, the network bright points (NBPs), which are pushed there by the photospheric convective flows to which they owe their proper motions. Inside the cell interiors lies the so called internetwork (IN), which is dominated by the granular motions and is characterized by small magnetic fields that move passively at the intergranular lanes.

When observed in $\mathrm{H} \alpha$ filtergrams, however, and especially in the center of the line, the quiet solar chromosphere loses its network appearance. Without loss of generality, the chromosphere in the $\mathrm{H} \alpha$ line center and nearby wavelengths can be defined as the collection of ubiquitous dark structures, known as mottles. Most of these are IN-spanning structures and it is believed that they outline magnetic canopies. They originate from the network boundaries and often form rosettes, centered on network bright point clusters. Only few quiet IN areas are not masked by such canopy structures. IN patterns, such as reversed granulation and grains, are not visible in $\mathrm{H} \alpha$ intensity images although there seems to be an $\mathrm{H} \alpha$ response in these phenomena (Rutten et al. 2008). It is worth saying that the $\mathrm{H} \alpha$ line spans a large height range from the photosphere, imaged at its wings, to the typical chromosphere at its line center (Leenaarts et al. 2006).

The distinct properties of the network and IN have been the subject of intense study. The NBPs exhibit long period oscillations, around 300 s or higher (Deubner \& Fleck 1989; Lites et al. 1993; Cauzzi et al. 2000), which are attributed to either magnetoacoustic waves (Deubner \& Fleck 1990; Lites et al. 1993) or to their erratic motions (Kneer \& von Uexküll 1986; von Uexküll et al. 1989). The same studies find that the most prominent periodicity in the IN is in the $3 \mathrm{~min}$ range $(5.5 \mathrm{mHz})$, while higher frequencies even up to $40 \mathrm{mHz}$ or more (De Forest 2004; Fossum \& Carlsson 2006) have been reported. The oscillations in the IN have been attributed to standing or evanescent acoustic waves. Along the mottles, oscillations with periods around 5 and 6 min have been detected (Tziotziou et al. 2004; Tsiropoula et al. 2009) and it seems that their dynamics differs from that of the IN 
they obscure. Although this is the behavior generally accepted for the oscillations in the aforementioned spatial regions, different results have also been reported (see the introduction of Tsiropoula et al. 2009), which may stem from the use of different diagnostics (different spectral lines and thus different heights of formation, filtergrams or slit images, different instruments etc.) and different methods (selection of regions and averaging).

Oscillatory motions in the solar atmosphere are of great interest, as they are thought to be signatures of wave energy carried outward from the photosphere, providing a considerable amount of heating to the upper atmosphere. Until recently, it was commonly understood that waves with frequencies below the acoustic cut-off frequency are evanescent and are trapped in the acoustic cavity of the Sun, while waves with frequencies above this can propagate freely in the atmosphere. However, several recent studies have reported upward traveling waves with frequencies below the acoustic cut-off. These waves propagate through "magneto-acoustic portals" that are created by areas of strong and significantly inclined (with respect to the surface gravity) magnetic fields (Jefferies et al. 2006). Much discussion has also been devoted to the "leakage" of photospheric oscillations into the chromosphere due to the lowering of the acoustic cut-off frequency within inclined flux tubes (De Pontieu et al. 2004). It is thus obvious that the different strengths and magnetic topologies of the magnetic fields are central in determining the differences in the wave properties and propagation characteristics.

A convenient way to parameterize the field strength is in terms of the plasma- $\beta$, the ratio of gas to magnetic pressure. The $\beta \sim 1$ layer, i.e. the region where the gas pressure and the magnetic pressure become comparable, partitions the atmosphere into contiguous volumes of high- $\beta$ and low- $\beta$ plasma and acts as a "magnetic canopy" for the waves (Rosenthal et al. 2002; Bogdan et al. 2003). The "canopy" provides a boundary wherein waves are reflected and refracted and plays a prominent role in influencing the mixing and conversion of waves. It is within the chromosphere that $\beta$ falls below 1 and magnetic forces start to gain control on the dynamics of the solar plasma resulting not only in mode conversion, refraction and reflection of waves, but also, together with the local thermodynamic properties, in the plethora of fine structures that characterize this part of the atmosphere.

Thus waves propagating from the photosphere to the chromosphere interact with the magnetic field and form complex patterns that are highly variable in space and time. This fact is not only a source of complications, but also means that wave properties can be used as a diagnostic tool for the properties of the atmosphere and also for the determination of the magnetic field geometry. Investigations of the spatial distribution of the oscillatory power have highlighted the presence of areas called "power halos" or "power aureoles", where the power is enhanced, and "magnetic shadows", where the power is suppressed. It has been found, from time series studies of photospheric Doppler velocities obtained with the Michelson Doppler Imager (MDI) and of intensities obtained in the chromospheric Ca II K line, that there is an enhancement of the oscillatory power at the period of 2-3 min in the surroundings of active regions (e.g. Braun et al. 1992; Brown et al. 1992; Hindman \& Braun 1998 1998; Thomas \& Stanchfield 2000; Muglach et al. 2005). It is called power halo and is associated, but it is not co-spatial, with magnetic field concentrations. This power halo is not observed in continuum intensity power maps. Krijger et al. (2001) confirmed the presence of power "halos" or "aureoles" in quiet Sun regions around the network patches observed in the UV data obtained with the Transition Region and Coronal Explorer (TRACE). On the other hand, Judge et al. (2001), using TRACE UV continua and Solar Ultraviolet Measurements of Emitted Radiation (SUMER) lines, have detected a deficit of acoustic power along with lower intensity values in the 2-3 min period range around the quiet Sun NBPs. This deficit, which they termed "magnetic shadow", had already been reported in previous studies (Kneer \& von Uexküll 1986; Cauzzi et al. 2000), but it has been further exploited in two-dimensional power maps in the past few years (Krijger et al. 2001; Muglach 2003; Muglach et al. 2005; Vecchio et al. 2007; Reardon et al. 2009). It is now generally accepted that the interaction of acoustic oscillations with the inclined magnetic field lines together with the location of the magnetic canopy are responsible for the formation of the power halos and magnetic shadows and may reflect common physical processes (McIntosh \& Judge 2001; McIntosh et al. 2003; Muglagh et al. 2005; Moretti et al. 2007).

In the present study we use observations obtained with the Dutch Open Telescope (DOT) in the $\mathrm{H} \alpha$ line to investigate the oscillatory behavior of the upper photosphere and chromosphere. As mentioned before, different wavelengths along the $\mathrm{H} \alpha$ profile provide information for different layers of the solar atmosphere. We therefore exploit these data to examine not only the horizontal distribution (comparing network and internetwork areas) of the oscillatory intensity and velocity power, but also the vertical one. The comparison between these different areas is in fact an indirect study of the effect of different magnetic topologies on the plasma dynamics. We also investigate the role of mottles (which trace the inclined magnetic field lines) in the formation of the magnetic canopy and the wave propagation between the upper photosphere and the chromosphere in a network region.

\section{Observations}

The observations used here are part of a set of observations obtained during a coordinated campaign in October 2007, which included space-born and ground-based instruments. For the present analysis we use only a time series of $\mathrm{H} \alpha$ observations of a quiet solar region, acquired by the Dutch Open Telescope (DOT) on La Palma on October 15. The observations were obtained with a Lyot filter, which transmits a tunable band of $0.025 \mathrm{~nm} F W H M$. The filter obtains bursts of 100 frames with exposure times of less than $20 \mathrm{~ms}$ at a regular cadence of $30 \mathrm{~s}$. A burst is split up into five different sub-bursts, each 20 frames long. After each sub-burst, the Lyot filter is shifted by $0.35 \AA$ in wavelength, thus obtaining observations at five wavelengths through the $\mathrm{H} \alpha$ profile, at $\pm 0.7 \AA, \pm 0.35 \AA$ and the line center. Observations at the near H $\alpha$ continuum at $6540 \AA$ (DOT's red continuum) were obtained as well. The individual frames of each sub-burst were speckle-reconstructed (to reduce the effects of atmospheric seeing) with the help of the red continuum burst, yielding one reconstructed image per sub-burst. The data cubes were then carefully aligned. The speckle reconstruction technique and other reduction steps are summarized in Rutten et al. (2004). The pixel size of the speckle-reconstructed images is $0.109^{\prime \prime}$. The common field-of-view is $84^{\prime \prime} \times 87^{\prime \prime}$ with its center located at the Sun's disk center. The solar region was continuously observed between 08:32 and 9:53 UT resulting in a 149-image sequence.

Sample images of the DOT observations covering the full FOV are shown in Fig. 1 together with a co-temporal, highresolution MDI magnetogram which reveals the magnetic topology of the observed quiet area. DOT H $\alpha$ observations render a 


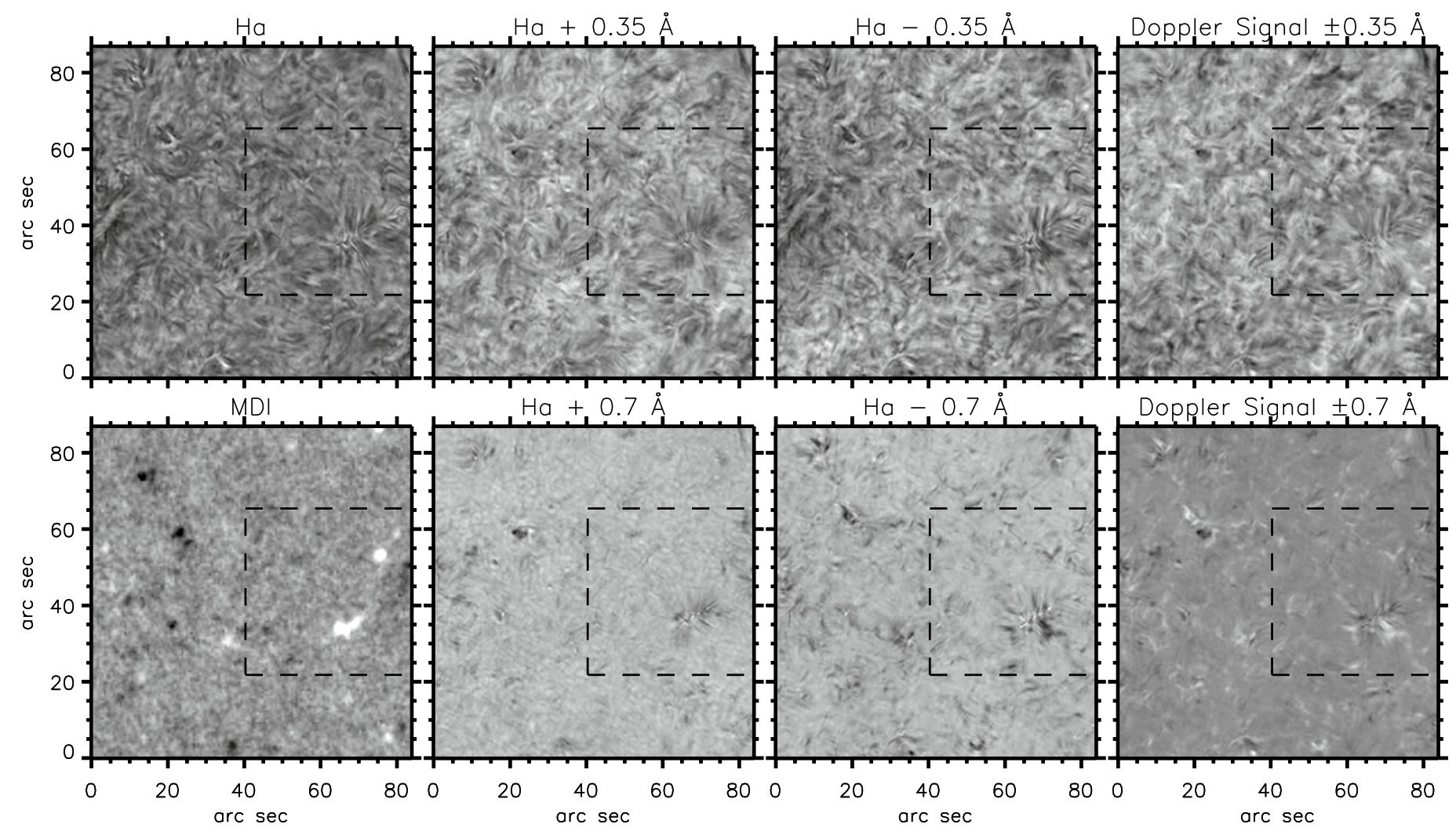

Fig. 1. Sample set of the full field-of-view of the DOT H $\alpha$ observations along with a co-temporal MDI magnetogram (second row, left image). The dashed square indicates the area that was further studied. Top row: $\mathrm{H} \alpha$ center, $\mathrm{H} \alpha+0.35 \AA$, $\mathrm{H} \alpha-0.35 \AA$ and Doppler signal at $\pm 0.35 \AA$ (scaled between \pm 0.35 ). Bottom row: MDI magnetogram (scaled between \pm 50 Gauss), $\mathrm{H} \alpha+0.7 \AA, \mathrm{H} \alpha-0.7 \AA$ and the Doppler signal at $\pm 0.7 \AA$ (scaled between \pm 0.4 ). For the definition of Doppler signal see Eq. (1).

tomographic view of the solar atmosphere from the photosphere $( \pm 0.7 \AA$ from $\mathrm{H} \alpha$ line center) to the chromosphere (H $\alpha$ line center). In $\mathrm{H} \alpha+0.7 \AA$ (Fig. 1, second row) we can clearly discern the granules as well as dark streaks which are parts of the elongated mottles seen better in the $\mathrm{H} \alpha$ line center. These emanate from the NBPs, which are grouped in clusters, mark the presence of photospheric magnetic elements related to strong magnetic fields and define the network boundaries. $\pm 0.35 \AA$ from $\mathrm{H} \alpha$ line center (Fig. 1, first row), the granules are not visible any more because $\mathrm{H} \alpha$ is formed at this wavelength at higher levels than the $\mathrm{H} \alpha$ wings. The image is slightly altered in the $\mathrm{H} \alpha$ line center, where even higher layers are sampled. At this level, the solar chromosphere shows a highly structured pattern with the mottles seen as absorbing features, which exhibit a variety of shapes and orientations and outline the local magnetic field topology.

\section{Data analysis}

Although the time span of the DOT data set is $\sim 1.5 \mathrm{~h}$, we selected a smaller subset of $30 \mathrm{~min}$ between 08:32 and 09:02 UT with a regular cadence, resulting in a 60 image sequence. Furthermore, we isolated a rectangular area of $43.6^{\prime \prime} \times 43.6^{\prime \prime}$ (called FOV hereafter, see Fig. 1) which contains a supergranular cell with a large rosette at the right lower boundary and a smaller one higher up, as well as internetwork.

From the $\pm 0.35 \AA$ and $\pm 0.7 \AA$ image pairs, two-dimensional Dopplergrams were calculated at each time step using the formula

$D S=\frac{I(+\Delta \lambda)-I(-\Delta \lambda)}{I(+\Delta \lambda)+I(-\Delta \lambda)}$, which gives the so-called "Doppler signal" (DS). According to this equation, a positive Doppler signal denotes an upward motion of absorbing material. All Doppler signals are relative to a zero reference defined by $D S=0$, which corresponds to the mean Doppler signal value of the whole FOV. This holds for each image as well as for the time-averaged one in Fig. 3. Of course, one must be aware that the derived values give only a parametric description of the actual velocity field but they can give a qualitative picture of upward and downward moving material (Tsiropoula 2000). Sample images of Doppler signals at $\pm 0.35 \AA$ and $\pm 0.7 \AA$ image pairs are shown in the last column of Fig. 1.

In order to compensate for the effect of the Doppler shift on the intensities taken on either side of the $\mathrm{H} \alpha$ profile, we use their wavelength average. This wavelength average, denoted hereafter by $\mathrm{H} \alpha \pm 0.35 \AA$, is the average intensity of the wavelengths $+0.35 \AA$ and $-0.35 \AA$ from the core (similarly $\mathrm{H} \alpha \pm 0.7 \AA$ ). Temporal average and minimum intensity values were calculated for the $\mathrm{H} \alpha$ line center, $\mathrm{H} \alpha \pm 0.35 \AA$ and $\mathrm{H} \alpha \pm 0.7 \AA$ at every pixel of the whole examined data set for the selected sub-field (Fig. 2). In all filtergrams the large rosette stands out at the lower right corner. In the average intensity images and in the rosette region darker areas represent places where frequent or persistent mottles occur. These elongated dark structures reduce the contrast of the average images at the cell interiors, leaving often a faint shadowy signature on them. In general, although it is in the rosette where these fibrilar structures are dominant, it is not difficult to maintain that the IN is a region unaffected by mottles (especially in $\mathrm{H} \alpha$ line center). 

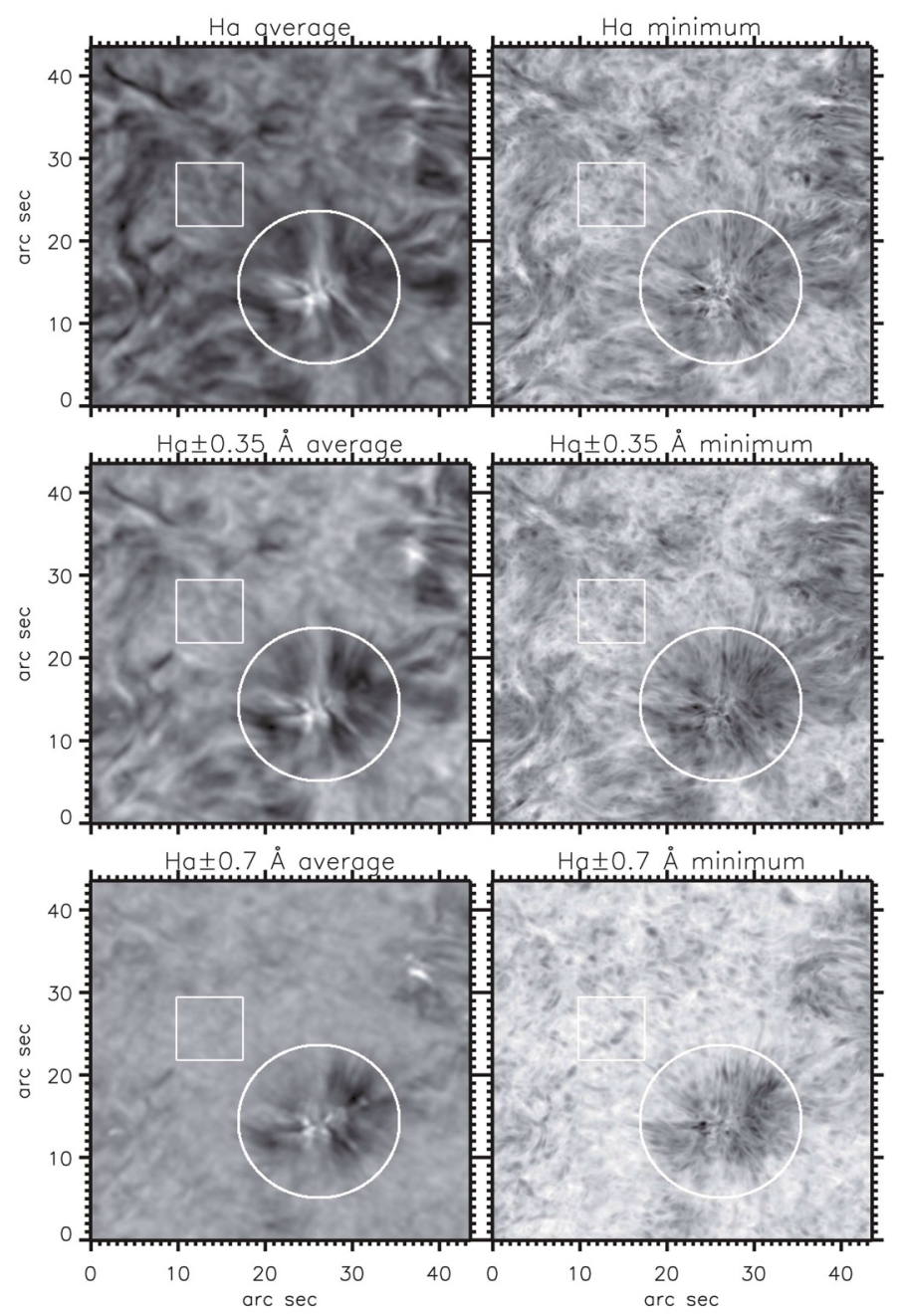

Fig. 2. Average (left column) and minimum (right column), with respect to time, of the $\mathrm{H} \alpha$ line center intensity and the wavelength averages $\mathrm{H} \alpha$ $\pm 0.35 \AA$ and $\mathrm{H} \alpha \pm 0.7 \AA$ (cf. Sect. 3). Shown is the subregion indicated in Fig. 1 by the dashed rectangle. The rectangles and circles denote the IN region and the rosette region, respectively, that were used for the derivation of Fig. 4 (cf. 4.2).

The minimum intensity maps of the FOV exhibit a finer texture than the average images and outline in a different fashion the different regimes that coexist at different atmospheric layers. Lower minimum intensity at each pixel of the image reflects absorption at that position of the $2 \mathrm{D}$ intensity map. This explains why we clearly see elongated dark (absorbing) structures at the minimum intensity images.

The time-averaged Doppler signal was also calculated for each pixel of the selected sub-field in $\pm 0.35 \AA$ and $\pm 0.7 \AA$ from the line center, which led to the construction of the time-averaged Doppler signal maps, shown in Fig. 3. The dark/bright features in these dopplergrams denote predominant downward/upward motion. There is a mixed pattern of upflows and downflows at the IN. This pattern disappears in the rosette region, leaving in its place a more organized fibrilar pattern, with elongated brightenings and darkenings that mark ascending and descending motions, respectively. There, especially at $\pm 0.35 \AA$ the dark areas are mainly situated close to the NBPs cluster, confirming previous findings (e.g. Tsiropoula et al. 1993), i.e. that downflows are dominant at the footpoints of the mottles, towards the center of the rosette. Several elongated brightenings
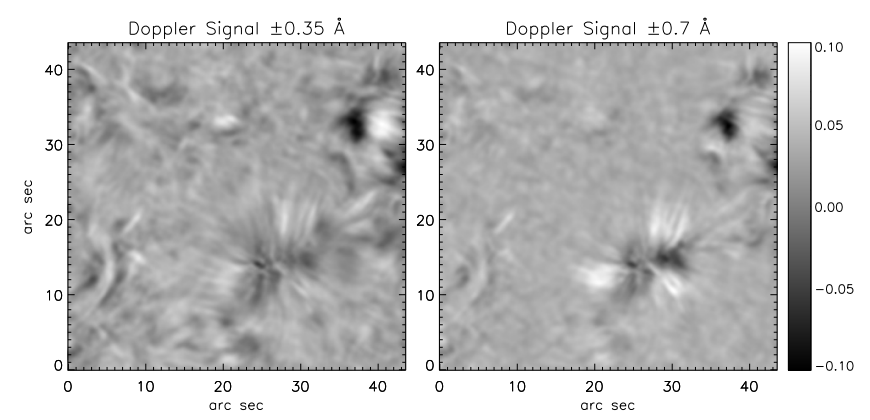

Fig. 3. Time averaged Doppler signal maps (see Eq. (1)) at $\pm 0.35 \AA$ (left column) and $\pm 0.7 \AA$ (right column) of the area shown in Fig. 1 .

that mark ascending motions are also visible at places where mottles occur.

The detection of oscillations in intensities and Doppler signals is carried out using the wavelet analysis, one of the most powerful and frequently used tools to investigate solar periodic phenomena. It is a two-dimensional transformation that also provides temporal information along with the detected periodicities. Moreover, a regular power spectrum comparable to that of a Fast Fourier transform (FFT) is calculated, which takes into account both the power and the duration of each oscillation in the signal. This is the global wavelet spectrum, comprising information that concerns the energy of the oscillations contained at each period. For our analysis we used the wavelet package freely distributed via the internet by Torrence \& Compo (1998) with a Morlet wavelet function as a basis for the transformation. Even though in the present study we use information only from the global wavelet spectrum which lacks any temporal information, we note that wavelet analysis is better suited for solar oscillations which show intermittency and non-stationarity.

Before applying the wavelet transform to our data we performed an FFT filtering to rule out periods greater than $600 \mathrm{~s}$, which reduce the power of smaller periods, due to the leakage of power towards high periods. We then performed a wavelet analysis on every pixel of the FOV and created maps of the average global wavelet power in three one-minute broad period bands, centered at 3,5 (the typical $p$-mode period) and $7 \mathrm{~min}$. This is done for the $\mathrm{H} \alpha$ line center intensity, the averaged intensities at the wings $( \pm 0.35 \AA$ and $\pm 0.7 \AA)$ and also for the respective Doppler signals.

\section{Results}

\subsection{Average power distribution of the $\mathrm{H} \alpha$ intensity and velocity}

In Fig. 4 we present the spatially averaged global intensity and Doppler signal power spectra as a function of period for two different areas observed in the FOV: one containing an IN area (dotted line) and the other a large rosette (solid line) that consists of several mottles. These areas are found inside the rectangle and the circle drawn in Fig. 2. Averaging was done by adding the power of each pixel and then dividing by the number of pixels that each area contains. Power values have been normalized to 1 . In the rosette region the power has a clear peak at $\sim 440 \mathrm{~s}$ in $\mathrm{H} \alpha$ intensities and in the Doppler signal at $\pm 0.7 \AA$ and it has a significant tail towards low periods. In the Doppler signal at $\pm 0.35 \AA$ the peak is at $320 \mathrm{~s}$ and has a broader distribution. These values are consistent with the findings of e.g. Tziotziou et al. (2004) and Tsiropoula et al. (2009), who reported a periodicity 

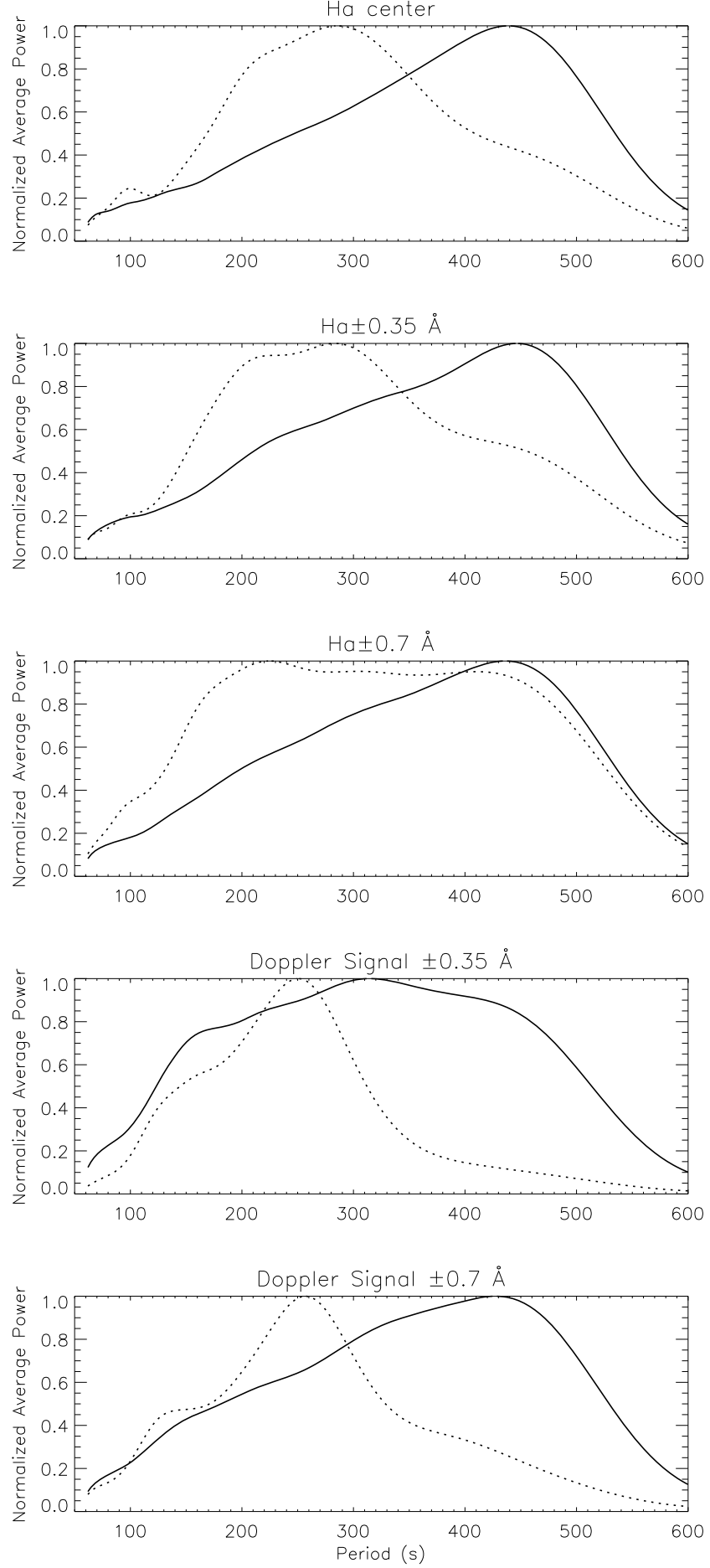

Fig. 4. Normalized average power of the intensity oscillations in the $\mathrm{H} \alpha$ line center and at $\pm 0.35 \AA, \pm 0.7 \AA$ (three first rows) and of the Doppler signals oscillations at $\pm 0.35 \AA, \pm 0.7 \AA$ (fourth and fifth row) for two representative regions of the FOV as a function of the period: an IN area (dotted line) and a rosette region (solid line). The first one is inside the rectangle and the second inside the circle drawn in Fig. 2.

in the 5-7 min range in both intensity and velocity variations of $\mathrm{H} \alpha$ mottles. This period contrasts to the clear 3 min periodicity, which is the chromospheric cut-off period, confirming the results of previous works (see e.g. the review by Rutten 1999).

In the IN area both Doppler signals show a power peak at $\sim 240 \mathrm{~s}$ and have a significant tail towards high periods.
Oscillations of the $\mathrm{H} \alpha$ intensities in $\mathrm{H} \alpha$ line center show a power peak at $\sim 290 \mathrm{~s}$, while for intensities in $\mathrm{H} \alpha \pm 0.35 \AA$ and $\mathrm{H} \alpha \pm 0.7 \AA$ the power is enhanced between 200 and $300 \mathrm{~s}$ and $200-450 \mathrm{~s}$ respectively. Although periodicities in the $3 \mathrm{~min}$ range have been usually attributed to IN regions, there are several works in which the authors find a power distribution peak at $\sim 300$ s with no enhanced power detectable in the 3 min range in lines formed in the chromosphere and transition region (see, e.g., Doyle et al. 1999; Cauzzi et al. 2000; Tsiropoula et al. 2009). Tsiropoula et al. (2009) pointed out that this result could arise because the IN regions are covered by inclined mottles and hence this period could be attributed to their presence.

From the power spectra of the intensities and Doppler signals, computed at each pixel of the FOV, we created 2-D power maps in the three period bands, centered at 3,5 and $7 \mathrm{~min}$. For each pixel, the power in each period band was computed by summing the power over all periods in the corresponding band. These maps are shown in Fig. 5 with logarithmic greyscaling. The three first rows are the power maps created from the $\mathrm{H} \alpha$ intensities in the line center, in $\pm 0.35 \AA$ and $\pm 0.7 \AA$, while the fourth and fifth row are the ones for the Doppler signals in $\pm 0.35 \AA$ and $\pm 0.7 \AA$, respectively. The left panels are power maps computed in the 3 min band, the middle in the 5 min band and the right in the $7 \mathrm{~min}$ band. The most striking feature in the power maps is that the distribution of power is structured and not random, coarsely following the morphology of the intensity distribution in the average and minimum images. More specifically, in the "mottle-free" IN there are bright and dark blobs which indicate places of enhanced power or a lack of it, respectively. At the rosette region and at all periods the power shows a remarkable filamentary distribution which is most probably related to the dark mottles that lie there (this is further explored below). Since the rosette has a more or less circular shape, we calculated the average power over expanding concentric circles, centered on a pixel considered as the rosette's center, marked as "+" in the first panel of the first row of Fig. 5. This will give us a measure of the azimuthally averaged distribution of the power as a function of the distance from the network. In the first panel of Fig. 5, concentric circles with 5", 10" and 15" radii have also been drawn. The variation of the azimuthally averaged power, as a function of distance from the center of the rosette is shown in Fig. 6, which is complementary to the power maps shown in Fig. 5.

In the following paragraphs we give a brief description of the power maps and the azimuthally averaged power distribution as shown in Figs. 5 and 6, respectively. It should be kept in mind that NBPs are found within 1 " -2 " from the "center" of the rosette while mottles reach up to distances of $99^{\prime \prime}-10^{\prime \prime}$. At greater distances, the average power refers more or less to the IN. We see that:

1. There is a sharp peak in the average power at $\sim 1^{\prime \prime}$ from the "center" of the rosette in the $\mathrm{H} \alpha$ line center (see first rows of Figs. 5 and 6), in all three period bands. This is the place where NBPs reside. The average power is then decreasing in all three period bands up to the distance of $9^{\prime \prime}$, an area covered by dark mottles. Up to this distance the average power is always higher at the $7 \mathrm{~min}$ period band and lower at the 3 min period band. Further away than $9^{\prime \prime}$ the average power at the $3 \mathrm{~min}$ and $5 \mathrm{~min}$ period bands is slightly increasing, while that corresponding to the $7 \mathrm{~min}$ band remains rather constant. The average power at the $5 \mathrm{~min}$ period band becomes slightly higher there than the one corresponding to 

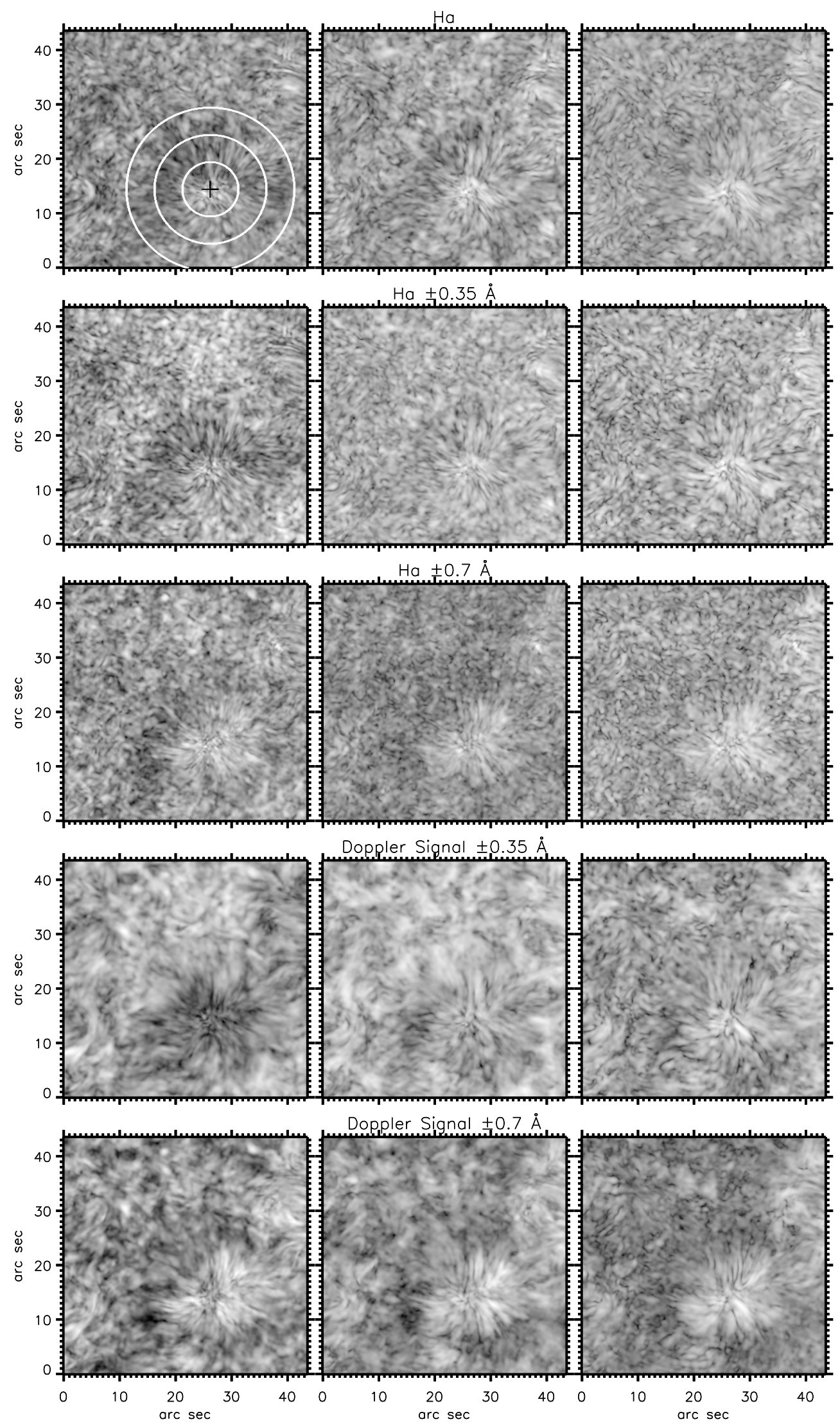

Fig. 5. Power maps (in a logarithmic grayscaling and scaled per panel) at the 3,5 and 7 min period bands (left, middle and right panels, respectively) in the $\mathrm{H} \alpha$ line center, $\mathrm{H} \alpha \pm 0.35 \AA$ and $\mathrm{H} \alpha \pm 0.7 \AA$ intensities and in $\pm 0.35 \AA$ and $\pm 0.7 \AA$ Doppler signals (from top to bottom). These figures provide also an insight on the vertical distribution of power at several heights. The black cross in the first panel marks the "center" of the rosette, whereas white circles denote distances of 5", 10" and 15" from this "center". The variations of power with distance shown in Fig. 6 have been calculated in respect to the position of the black cross. 


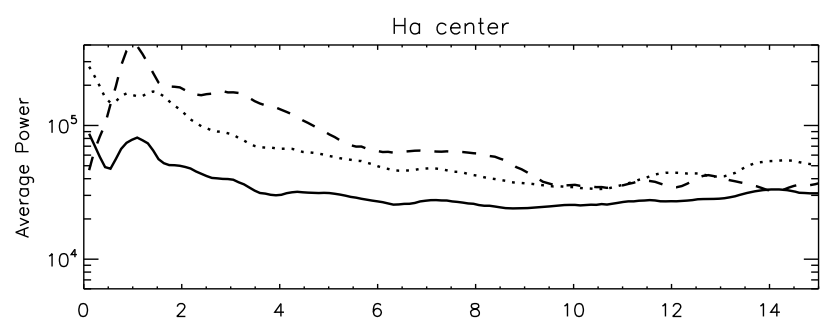

$\mathrm{Ha} \pm 0.35 \AA$
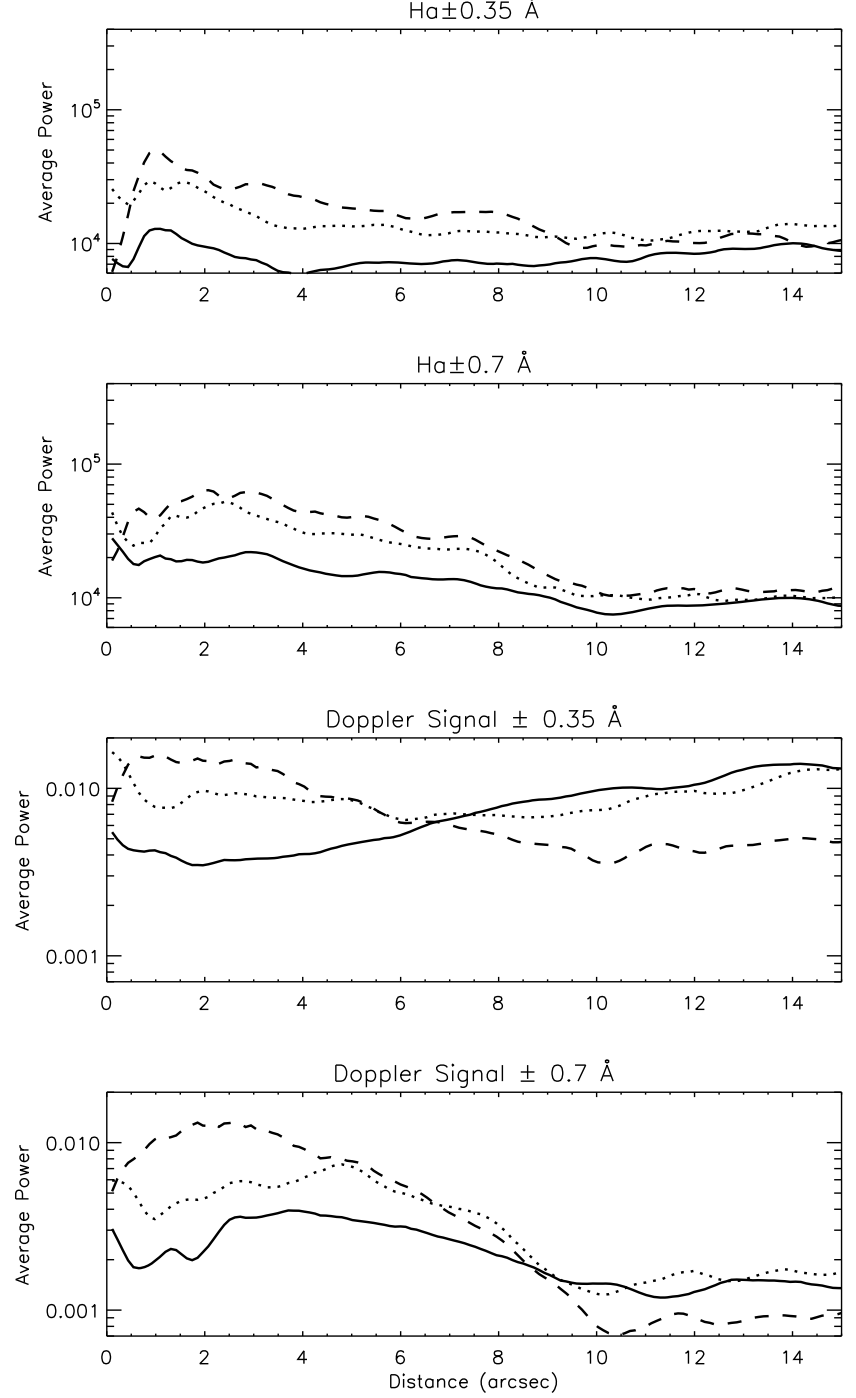

Fig. 6. Variation of the azimuthally averaged power as a function of distance from the center of the rosette, marked as "+" in Fig. 5 (see text for details), at $3 \mathrm{~min}$ (solid), $5 \mathrm{~min}$ (dotted) and $7 \mathrm{~min}$ (dashed) period bands in the $\mathrm{H} \alpha$ line center, $\mathrm{H} \alpha \pm 0.3 \AA$ and $\mathrm{H} \alpha \pm 0.7 \AA$ intensities and in $\pm 0.35 \AA$ and $\pm 0.7 \AA$ Doppler signals (from top to bottom). Note that the NBP's are located roughly within 1 " -2 " from the "center" of the rosette while mottles lie between $2^{\prime \prime}-10^{\prime \prime}$.

the other two bands. It should be mentioned that few mottles are long enough to reach distances beyond $9^{\prime \prime}$.

2. The power distribution of the intensity oscillations in $\pm 0.35 \AA$ is similar to that of $\mathrm{H} \alpha$ at the line center (see second row of Figs. 5 and 6 ). The peak of the power at $\sim 1^{\prime \prime}$ related to the NBPs is also observed in all three period bands. The average power of the intensity oscillations at the $3 \mathrm{~min}$ period band is lower than that of the other two bands, while the corresponding power at the 7 min period band is again higher up to a distance of 9 " from the "center" of the rosette and then becomes lower than the power of the 5 min band. The remarkable behavior to notice is the abrupt decrease of the power after the peak at $1^{\prime \prime}$ which becomes more pronounced at the $3 \mathrm{~min}$ period band, but is also prominent at the $5 \mathrm{~min}$ period band. The power distribution attains a minimum at $4^{\prime \prime}$ and is clearly related to the inner parts of the mottles. Then, at the 3 min period band the power increases continuously outwards up to $\sim 14^{\prime \prime}$, while it remains constant at the $5 \mathrm{~min}$. The power of the Doppler signal oscillations at $\pm 0.35 \AA$ (see fourth row of Figs. 5 and 6) shows a suppression in the area of NBPs which is more obvious at the 5 min period band, but it is also apparent at the $3 \mathrm{~min}$ band. The averaged power at the 3 min period band has a minimum at $2^{\prime \prime}$ and then it increases continuously outwards. Beyond $8^{\prime \prime}$ it becomes higher than the power in the other two bands. The power at the $5 \mathrm{~min}$ period band decreases beyond $2^{\prime \prime}$ up to $6^{\prime \prime}$, an area clearly related to the inner parts of the mottles, and then increases outwards. At the $7 \mathrm{~min}$ band there is a flat peak of the power between $1^{\prime \prime}$ and $4^{\prime \prime}$, an area that contains both the NBPs and the footpoints of the mottles. Beyond this distance the power decreases continuously outwards till $10^{\prime \prime}$ and then increases, remaining lower than the power in the other two bands after $6^{\prime \prime}$.

3. In $\mathrm{H} \alpha \pm 0.7 \AA$ (see third rows of Figs. 5 and 6 for the average power distribution of the intensity oscillations and fifth rows of the same figures for the average power distribution of the Doppler signal oscillations) the distribution of the average power in both intensity and Doppler signal is similar. At the $3 \mathrm{~min}$ and $5 \mathrm{~min}$ period bands there is a suppression of the power at the central part of the rosette related to the NBPs. After this power suppression, there is a power enhancement in all three period bands (less pronounced in the $3 \mathrm{~min}$ period band), more extended spatially and clearly related to the fibrilar structures. The power peak of the intensity oscillations in all three period bands is at $\sim 2.5^{\prime \prime}$ from the center of the rosette, while the corresponding peak of the Doppler signal at the $7 \mathrm{~min}$ period band is at $2^{\prime \prime}$, at the $5 \mathrm{~min}$ band at $5^{\prime \prime}$ and at the 3 min band at $4^{\prime \prime}$. The power in all three period bands decreases smoothly up to $10^{\prime \prime}$. Beyond $10^{\prime \prime}$ the power in all three bands either increases slightly or remains constant.

We will attempt to interpret the observational results that were derived by our analysis and described above, in the discussion section. We can make the following remarks though:

- As mentioned before, few mottles are long enough to reach distances beyond $9^{\prime \prime}$, hence the decrease of the 7 min power beyond that distance in all panels of Fig. 6 indicates that the corresponding $7 \mathrm{~min}$ oscillations are strongly related to their presence. This could also explain why the power within the first $9^{\prime \prime}$ especially at higher periods, exceeds the power at the adjacent IN which starts beyond 9". The former probably relates to the propagation/refraction of waves in/from mottles, while the latter relates to lower amplitude oscillations that do not reach chromospheric heights.

- The increase of the extent of the dark patches in the power maps as we move from lower to higher layers (i.e. from $\mathrm{H} \alpha$ $\pm 0.35 \AA$ to line center) reveals the expansion of the magnetic field flux tubes that emanate from the network areas.

We must note that the same behavior as outlined above for the large rosette is also exhibited by the network region at the top right of the FOV. The rosette is much less extended there and the 


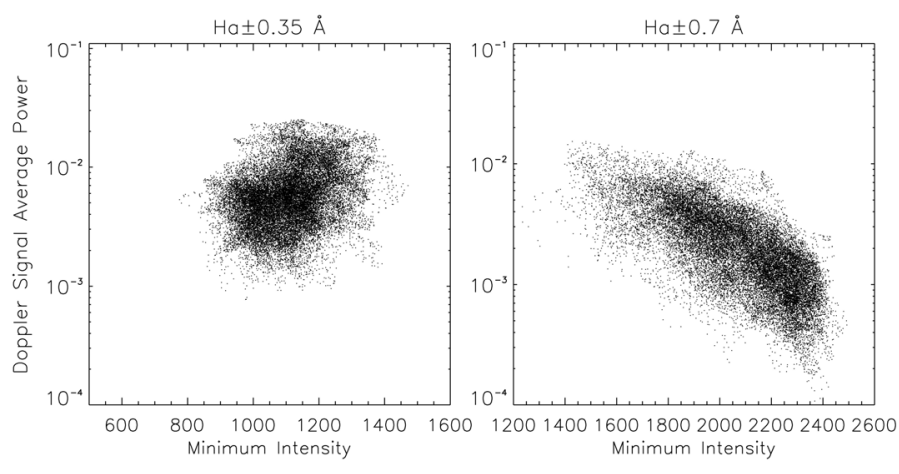

Fig. 7. Scatter plots of the average power of Doppler signal variations in a logarithmic scale vs. the $\mathrm{H} \alpha \pm 0.35 \AA$ minimum intensity (left) and the $\mathrm{H} \alpha \pm 0.7 \AA$ minimum intensity (right) at the $3 \mathrm{~min}$ period band in the rosette region between $2^{\prime \prime}$ and $9^{\prime \prime}$ from its center, which is noted by the black cross in Fig. 5.

same is true for the subsequent suppressions and enhancements of the power.

\subsection{Power distribution and its relation to mottles}

The discrimination between the different structures observed in the FOV using an intensity threshold is not an easy task; most pixels switch between categories as time elapses, and an absorbing feature is not necessarily at the same position in all five wavelengths of the $\mathrm{H} \alpha$ line profile. An important question to answer is whether the power enhancement or suppression within the rosette region, discussed in the previous section, is in fact related to the dark mottles or not, even though it has a fibrilar structure. We seek evidence for a correlation (or lack of it) through a comparison of the power with the intensity. As can be seen in Fig. 2, the mottles appear darker than the background and have coarsely a lower minimum intensity value.

In Fig. 7 we show scatter plots between the average power of the oscillations of the Doppler signal and the minimum intensity in $\mathrm{H} \alpha \pm 0.35 \AA$ (left) and $\mathrm{H} \alpha \pm 0.7 \AA$ (right) in the area centered on the rosette region and contained between $2^{\prime \prime}$ and $9^{\prime \prime}$ where the power enhancement and suppression are observed. We choose the Doppler signal oscillatory power because it shows a more pronounced power enhancement and suppression. In the left scatter plot lower power values correspond to lower minimum intensity values, while in the right one the inverse is true. The weaker correlation that is exhibited at the left scatter plot is probably due to the lower contrast between mottles and the internetwork at $\pm 0.35 \AA$, since this wavelength is closer to the core of the $\mathrm{H} \alpha$ profile.

Of course, it is not expected a precise relationship between the $\mathrm{H} \alpha$ wing absorption and the oscillatory power suppression/enhancement, nonetheless, these scatter plots clearly indicate that power suppression at $\pm 0.35 \AA$ and enhancement at $\pm 0.7 \AA$ shows up preferentially at the positions of mottles.

\subsection{Phase differences and coherence}

The propagation of waves through different layers of the solar atmosphere was studied using a phase difference analysis. We used a cross wavelet transform (Torrence \& Compo 1998) and we compute the coherences and phase differences between the Doppler signals at $\pm 0.35 \AA$ and at $\pm 0.7 \AA$. The former represents velocities at a chromospheric level and the latter velocities at the upper photosphere. The phase differences between intensities are not discussed here because they will be the subject of a follow-up paper. The phase difference for every pixel of the FOV was calculated in the same way as the average power, i.e. the phase differences for every pixel within each of the 3 period bands were averaged, thus assigning an average phase difference within the band to each pixel. An average coherence value for the respective period band as well as a standard deviation were also derived. The 2D coherence and phase difference maps of the FOV in the three period bands are shown in Fig. 8. In the coherence maps (Fig. 8, first row) brighter pixels correspond to a higher coherence while in the phase difference maps (Fig. 8, second row) positive phase differences correspond to upward propagation. In these maps white pixels correspond to phase differences with a coherence of less than 0.5 and a standard deviation greater than $10^{\circ}$. With the latter criterion we try to avoid any misinterpretation that might result from averaging a wide range of phase differences inside the band. We note however that areas where the standard deviation of the average phase difference is greater than $10^{\circ}$ are roughly within the areas of lower coherence.

In Fig. 9 we have plotted the histogram of the phase differences obtained in each one of the three bands in the rosette and the IN region, included in the circle and square drawn in Fig. 8 (see also Fig. 2). In these histograms, phase differences of oscillations with a low coherence $(<0.5)$ have been neglected. At the IN (Fig. 9, right panel) 3 min oscillations dominate and phase differences indicate predominant upward propagation (positive phase differences). The latter is also true for the $5 \mathrm{~min}$ oscillations. In the evanescent regime (7 min oscillations), most of the internetwork exhibits small absolute phase differences (between \pm 20 degrees), as is expected for standing or evanescent waves. Larger negative phase differences have been attributed to downward propagating gravity waves (Cauzzi et al. 2000; Krijger et al. 2001; Rutten \& Krijger 2003).

Inside the rosette region (see Fig. 8 and left panel of Fig. 9) the phase differences are positive at the $3 \mathrm{~min}$ band, while the coherence in most of the pixels is lower than 0.5 . This is most probably due to the lower power of the Doppler signal at $\pm 0.35 \AA$ (see also Fig. 6) and points to the fact that these waves do not propagate vertically upward due to the inclined magnetic flux tubes. At the 5 min period band, phase differences have both positive and negative values, although slightly more positive than negative. In comparison with the $3 \mathrm{~min}$ band, more pixels have a coherence higher than 0.5 due to the higher average power. Again positive phase differences may indicate waves which are not impeded by the inclined magnetic fields and travel upward, while downward directed waves may be the result of the reflection of acoustic oscillations at the inclined magnetic fields. At the $7 \mathrm{~min}$ period band, there is fibrilar structure which is also apparent in the power maps and may point to mottles as the guides of wave propagation both upward and downward, depending on their inclination. We must note that only vertical propagation can be examined through this kind of analysis and no conclusions concerning the transversal propagation along mottles can be safely drawn. However, this is another indication that the $7 \mathrm{~min}$ period is strongly related to the presence of mottles, as was already mentioned. At the $7 \mathrm{~min}$ period band in the rosette region, phase differences are almost equally distributed around zero, with a slight tendency towards positive values. In the rosette region oscillations have coherence values higher that 0.5 in almost every pixel. At the $5 \mathrm{~min}$ and $7 \mathrm{~min}$ period bands the negative phase differences in several parts of the rosette are an indication that part of the acoustic oscillations cannot propagate upward 

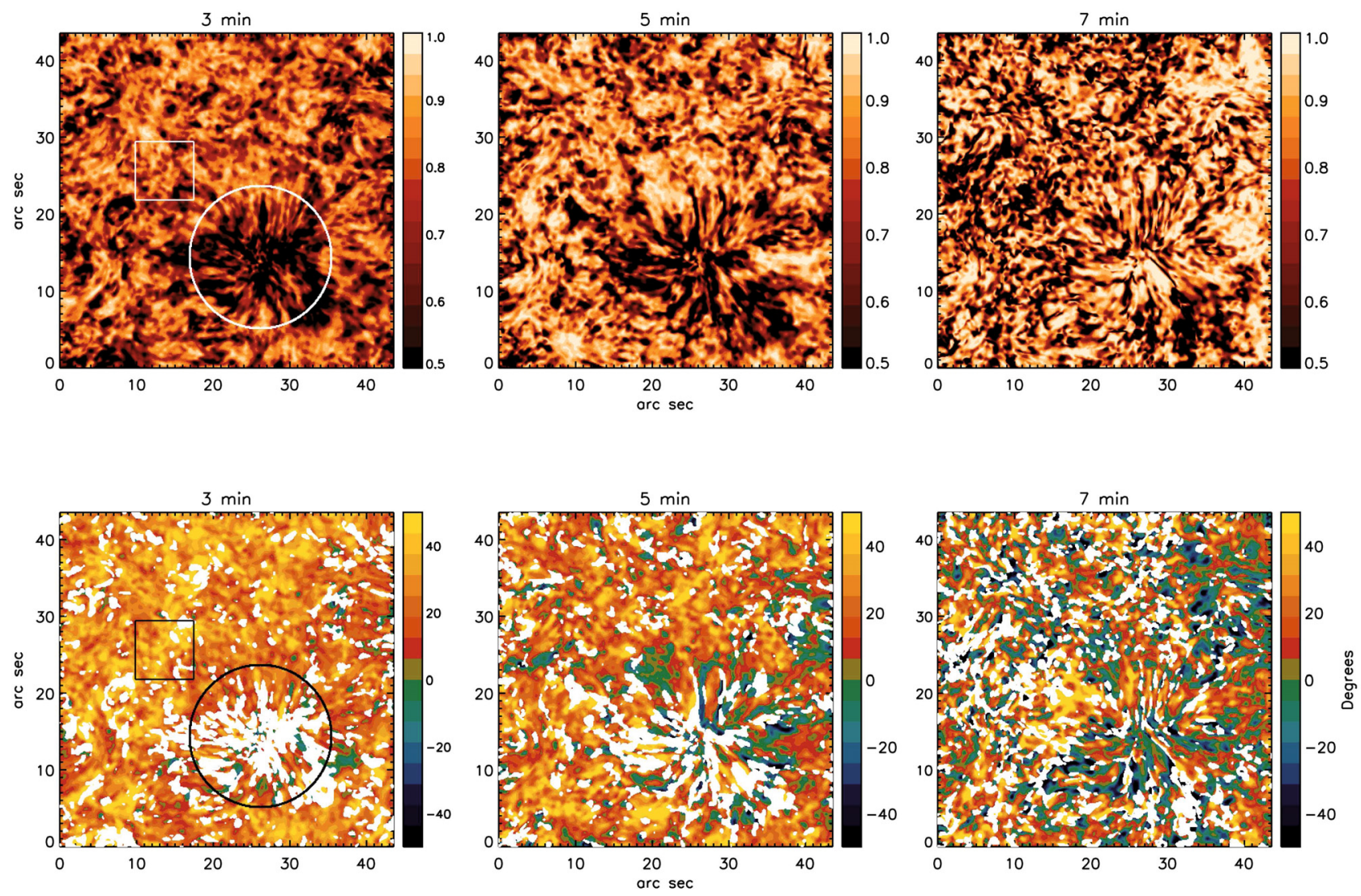

Fig. 8. Coherence (first row) and phase difference (second row) maps between Doppler signals at $\pm 0.35 \AA$ and $\pm 0.7 \AA$ for the 3 min, 5 min and 7 min oscillations (first, second and third column respectively). In the second row, white areas represent oscillations with a coherence lower than 0.5 (darker areas at the maps of first row) and/or a standard deviation larger than $10^{\circ}$. Positive phase difference indicates upward propagating disturbances and vice versa. In the first panel of each row the drawn circles and rectangles mark the rosette and IN region for which the histograms of Fig. 9 have been derived. (In color in the electronic edition.)

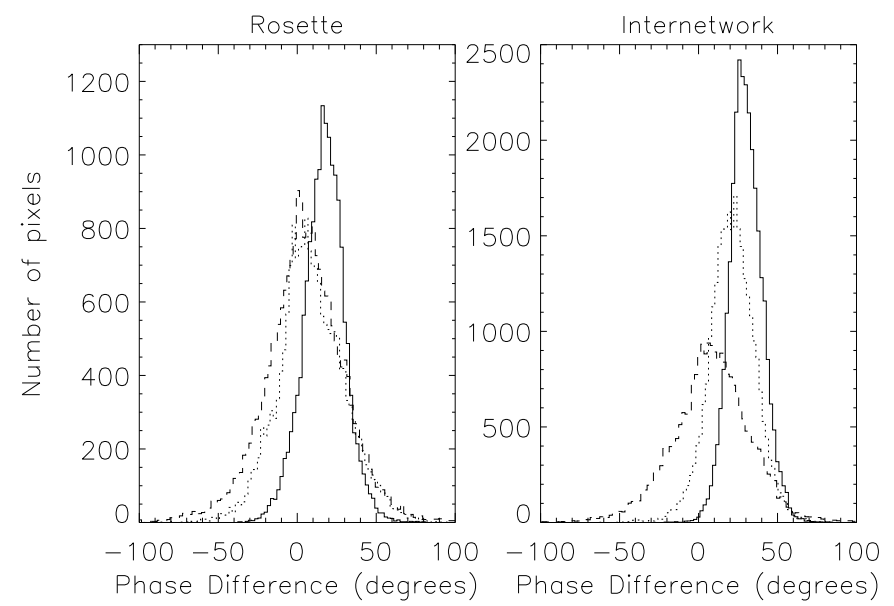

Fig. 9. Histograms of the phase differences at the $3 \mathrm{~min}$ (solid line), 5 min (dotted line) and 7 min (dashed line) period bands in the rosette region (left) and the IN (right) found within the circle and rectangle, respectively, drawn in Fig. 8.

because of the presence of the inclined mottles, which can reflect part of the waves.

\section{Discussion and conclusions}

We have presented an analysis of time sequences of $\mathrm{H} \alpha$ filtergrams obtained by the DOT in five wavelengths along the $\mathrm{H} \alpha$ line profile as well as of Doppler signals derived from wavelengths on either side of the $\mathrm{H} \alpha$ profile. In general, the $\mathrm{H} \alpha$ line center marks the location of the "typical" chromosphere, forming at heights above $1500 \mathrm{~km}$, while the regions sampled at the far wings are well below the temperature minimum (Vernazza et al. 1981). In this direction point also the results obtained by the more recent calculations of Leenaarts et al. (2006), who combine MHD simulations together with non-LTE radiative transfer. They have shown that while the $\mathrm{H} \alpha$ line wing at $0.84 \AA$ is formed at the photosphere, the wings at $0.34 \AA$ contain a significant contribution from heights greater than $1000 \mathrm{~km}$ above the photosphere, as well as a photospheric contribution. It is therefore obvious that the $\mathrm{H} \alpha$ line profile is a powerful diagnostic tool since it provides information from the photospheric up to the chromospheric layers. In our $\mathrm{H} \alpha$ observations this is partially revealed from the fact that $\mathrm{H} \alpha \pm 0.35 \AA$ filtergrams show no granulation and resemble the $\mathrm{H} \alpha$ line center images. What we actually see comparing the quiet Sun from the $\mathrm{H} \alpha$ wings to line center is a change of layers, going from the photospheric one with clear granulation to the chromospheric where the IN is more or less covered by the dark mottles that emanate from network boundaries. These dark features play a crucial role in shaping the chromospheric dynamics. Their ubiquitous presence can explain our finding concerning the shift of the IN periods from the $3 \mathrm{~min}$ range to $5 \mathrm{~min}$ (which is the period observed in mottles), when moving from the lower to the higher atmospheric layers.

Our 2D power maps of the intensities and Doppler signals and also the $2 \mathrm{D}$ phase difference maps of the Doppler signals 
show at all examined periods a strikingly filamentary structure in the rosette region. This region consists of several dark mottles that most likely outline the local magnetic field topology. We have found power enhancement of the intensity and of the Doppler signal at $\pm 0.7 \AA$ at all three examined period bands around the network boundaries and, more specifically, over dark mottles. At the same wavelength, there is power enhancement at the $3 \mathrm{~min}$ and $5 \mathrm{~min}$ period bands over the NBPs. Such power enhancements have already been observed at 3-5 min periods in active regions around plages (Braun et al. 1992; Brown et al. 1992; Hindman \& Braun 1998; Thomas \& Stanchfield 2000; Muglach 2003; Muglach et al. 2005) and in quiet regions around the network patches (Krijger et al. 2001; Muglach et al. 2005) and have been called "power halos" or "aureoles".

In contrast to the power halo observed at the photospheric level, there is a clear suppression of the power of the $\mathrm{H} \alpha$ $\pm 0.35 \AA$ Doppler signal and of the $\mathrm{H} \alpha$ line center and $\mathrm{H} \alpha$ $\pm 0.35 \AA$ intensities, more intense at the 3 min period band, but also present at the $5 \mathrm{~min}$ and $7 \mathrm{~min}$ period bands. On the contrary, at the same wavelengths there is a power peak in the area of the NBPs in the intensities in all three period bands, while in the Doppler signal this is the case only in the $7 \mathrm{~min}$ period band. The power suppression points to the magnetic shadows detected by recent studies (Judge et al. 2001; McIntosh \& Judge 2001; Krijger et al. 2001; Vecchio et al. 2007) in network regions. This has also been noted previously by Kneer \& von Uexküll (1986) who reported significantly reduced acoustic power of the $\pm 0.45 \AA \mathrm{H} \alpha$ wings at the network boundaries, prominent around the $3 \mathrm{~min}$, but less intense around $5 \mathrm{~min}$. Most of the above cited authors note that power suppression does not seem to be related directly to the NBPs, but to the region adjacent to them. From our high resolution DOT $\mathrm{H} \alpha$ data and from the Interferometric Bidimensional Spectrometer (IBIS) data in the Ca II $8542 \AA$ and Ca II K chromospheric lines (Vecchio et al. 2007; Reardon et al. 2009) it is obvious that there is a clear correlation of the power enhancement or suppression with the dark mottles surrounding the NBPs. Indeed, as we have shown in this work, the power suppression as well as the power enhancement are clearly related to dark mottles. In the UV continua of TRACE and the SUMER lines, examined by previous studies (Judge et al. 2001; McIntosh \& Judge 2001), these features are not resolved due to the lower spatial resolution of both instruments and the width of TRACE filters.

The above reported observational results suggest that the mottles which surround the NBPs and their magnetic fields are related to the enhancement (power halo) or suppression (magnetic shadow) of the oscillatory power observed in the photosphere and chromosphere in network areas. We suggest the following scenario to explain the observations:

1. In the central part of the rosette, where the magnetic elements mark the positions of mainly vertical flux tubes, there is a power peak at all wavelengths and all three period bands (with an exception of both the Doppler signals period and the $\mathrm{H} \alpha \pm 0.7 \AA$ in the $3 \mathrm{~min}$ and $5 \mathrm{~min}$ ). The power is higher in the $7 \mathrm{~min}$ period band and lower in the $3 \mathrm{~min}$ period band. How can these results be explained? At the photospheric level there are acoustic waves (the global $p$-modes). These waves are largely evanescent: their periods are well above the cut-off period in the upper photosphere and the lower chromosphere. It has long been thought that acoustic waves propagating vertically in the quiet solar atmosphere change their dominating period with height, from $5 \mathrm{~min}$ in the photosphere to $3 \mathrm{~min}$, which is the cut-off period in the chromosphere (Bel \& Leroy 1977). However, numerous observations suggest that there is no such change for waves observed in network regions at chromospheric heights. Roberts (1983) propose in a theoretical analysis that an increase in the acoustic cut-off period can be produced by radiative energy losses in thin flux tubes. This analysis has been further explored by means of 2D numerical simulations by Khomenko et al. (2008). They concluded that the efficiency of the energy exchange by radiation in the solar photosphere can lead to a significant increase of the cut-off period and may allow for the vertical propagation of the 5 min waves into the chromosphere. These theoretical results can explain our observations in the magnetic elements area, i.e. that increased power is observed not only in the $3 \mathrm{~min}$, but also in the $5 \mathrm{~min}$ and even in the $7 \mathrm{~min}$ period range.

2. As network flux tubes expand with height, their field lines get more and more inclined until they either become horizontal or encounter field lines from other flux tubes and thus form the so-called magnetic canopy. This is an important transition layer, where the plasma $\beta$ is of order unity and separates the weakly magnetized photosphere from higher regions. It is in this layer where waves interact with the magnetic field and wave mode conversions or reflections occur. The behavior of the observed power distribution as shown in Figs. 5 and 6 can now easily be explained by means of the mottles (inclined magnetic flux tubes), the existence of the magnetic canopy and mode reflection/conversion of the waves. The power distribution of the intensity and of the Doppler signal at $\pm 0.35 \AA$ clearly shows how the propagation of the waves depends on the magnetic field topology. It seems that the power suppression ("magnetic shadow") observed in the $3 \mathrm{~min}$ and $5 \mathrm{~min}$ period bands, in the layer where $\mathrm{H} \alpha \pm 0.35 \AA$ is formed and in the area covered by the mottles, marks the presence of the "magnetic canopy". At this layer at least a part of the waves is reflected by the overlying magnetic fields, while another part propagates upward. To what extent waves are reflected depends on the local properties of the atmospheric layer and on the angle between the waves and the magnetic field. The reflected waves return to the photosphere and increase the oscillatory power locally, thus forming (or increasing) the photospheric power halos. This effect can explain the power enhancement of the intensity and the Doppler signal at $\pm 0.7 \AA$ (i.e. in a layer formed in the photosphere). The same behavior of the power distribution at the $0.35 \AA$ wavelength is observed in the center of the $\mathrm{H} \alpha$ line (i.e. a power decrease in the mottles' area). It seems that part of the waves, which are not reflected in the magnetic canopy, are propagating upward. It should also be noticed that not only waves in the $3 \mathrm{~min}$ band (the acoustic cut-off), but also in the $5 \mathrm{~min}$ and the $7 \mathrm{~min}$ bands propagate at the $\mathrm{H} \alpha$ formation layer.

How can the evanescent 5 min oscillations propagate up to chromospheric heights? Much discussion has been devoted to the "leakage" of photospheric oscillations and flows into the chromosphere due to the increase of the acoustic cut-off period within inclined magnetic fields. These analyses have been based on observations of fibrils in active regions (De Pontieu et al. 2004), but also on quiet Sun mottles (Tsiropoula et al. 2009; see also Suematsu 1990). Recent results reported by Jefferies et al. (2006) show that a sizable fraction of the photospheric acoustic power at periods above the acoustic cut-off might propagate to higher layers within and around the magnetic network elements. They also argue that "leakage" of these high-period oscillations 
positioned within and around the network into the chromosphere through "magneto-acoustic portals" might provide a significant source of the energy necessary for heating the quiet chromosphere. The above scenario is also supported by the calculated phase differences between the Doppler signals at $\mathrm{H} \alpha \pm 0.35 \AA$ and $\mathrm{H} \alpha \pm 0.7 \AA$. In the rosette region, we have found upward propagating waves in the 3 min period band in the pixels with a coherence higher than 0.5 , although in most of the pixels the coherence is lower than 0.5 due to the lower power (suppression) of the Doppler signal at $\pm 0.35 \AA$. At the $5 \mathrm{~min}$ and $7 \mathrm{~min}$ period bands both positive and negative phase differences are obtained, more positive in the former than in the latter. The positive phase differences point to upward propagating waves, the negative ones to waves propagating downwards due to their reflection by the inclined mottles. The peak around $0^{\circ}$ at the $7 \mathrm{~min}$ period band marks the presence of standing waves that are formed close to the canopy due to the interference of the refracted waves with the upward propagating waves.

As noted in Jefferies et al. (2006), the net mechanical energy flux provided by the upward high period channeled waves could provide a considerable fraction of the energy needed to balance the radiative chromospheric losses. On the other hand, observational data seem to suggest that most likely magnetic reconnection is involved in dark mottles emanating from the network boundaries due to the bipolar nature of the flux cancellation process of opposite polarity magnetic fields swept to the network boundaries by the supergranular flow (Tsiropoula et al. 1993; Tziotziou et al. 2003). Tsiropoula \& Tziotziou (2004), based on the suggestion of magnetic reconnection as the driving mechanism of mottles, obtain estimates of their role in the mass balance and energy budget of the solar atmosphere, neglecting however the role of waves, due to the lack of corresponding information. With the present results it seems quite possible that both mechanisms (e.g. magnetic reconnection and upward propagating waves) are at work. This leads us to suggest that maybe the chromosphere and the corona are in fact heated by a wide variety of mechanisms that each can provide a percentage of the required heating rate. In this sense the relative contributions of the various phenomena related to mottles have to be determined quantitatively. The above statements make the study of mottles very attractive.

From the 2D power and phase difference maps it is revealed that different behavior is encountered in the IN and the network. This diversity is beyond any doubt due to the magnetic field geometry which seems to play an important role in wave propagation. The scenario of an acoustic dominated sub-canopy chromosphere that pummels and interacts with an overlying magnetic one is very appealing (Rutten et al. 2008). The latter is usually assumed to be situated at $\sim 1000 \mathrm{~km}$. We believe that the $\mathrm{H} \alpha \pm 0.7 \AA$ formed at the photosphere together with the $\mathrm{H} \alpha$ $\pm 0.35 \AA$ wings, formed at that height, show in the most pronounced way the transition from the acoustic sub-canopy chromosphere (also termed as "fluctosphere") to the magnetic "typical" $\mathrm{H} \alpha$ chromosphere. In fact, $\mathrm{H} \alpha \pm 0.35 \AA$ resembles the line center more than the further wings of $\mathrm{H} \alpha$. More specifically, it seems that the $\beta=1$ layer is below the formation height of the $\mathrm{H} \alpha \pm 0.35 \AA$ and this can explain the reduced chromospheric power at the formation height of this wavelength as well as the enhanced power in the $\mathrm{H} \alpha \pm 0.7 \AA$.

The above stated results will be further investigated in combination with simultaneous, co-spatial time sequences of filtergrams taken by TRACE at $1550 \AA 1600 \AA$ and $1700 \AA$ and MDI high-resolution magnetograms obtained during our campaign.
This will allow us to make one-to-one comparison of the power distributions and examine their changes with height. Phase differences and coherence will be calculated to get an insight into the different types of waves. The 3D magnetic field topology will be determined using the simultaneous observations obtained by the Spectropolarimeter (SP) on-board Hinode. The combination of these data will yield a powerful diagnostic suite for studies of solar wave phenomena in network regions, providing a link to the understanding of wave mode conversion and dissipation and ultimately to the heating mechanism of the chromosphere and the corona and will accurately allow the mapping of the important $\beta=1$ layer.

Acknowledgements. The DOT is operated by Utrecht University at the Spanish Observatorio del Roque de los Muchachos of the Instituto de Astrofísica de Canarias. The authors thank P. Sütterlin for the observations and R. Rutten for the data reduction. They also thank the International Space Science Institute (ISSI) in Bern, Switzerland, for the hospitality provided to the members of the team on "Solar small-scale transient phenomena and their role in coronal heating" as well as the members of the team for fruitful discussions.

\section{References}

Bel, N., \& Leroy, B. 1977, A\&A, 55, 239

Bogdan, T. J., Carlsson, M., Hansteen, V., et al. 2003, ApJ, 599, 626

Braun, D. C., Duvall, T. L., Jr., Labonte, B. J., et al. 1992, ApJ, 391, L113

Brown, T. M., Bogdan, T. J., Lites, B. W., et al. 1992, ApJ, 394, L65

Carlsson, M., \& Hansteen, V., 2005, The Dynamic Sun: Challenges for Theory and Observations, Proceedings of the 11th European Solar Physics Meeting, ed. D. Danesy, S. Poedts, A. De Groof, \& J. Andries, 11-16 September, Leuven, Belgium, published on CDROM., 16.1, ESA SP-600

Cauzzi, G., Falchi, A., \& Falciani, R. 2000, A\&A, 357, 1093

DeForest, C. E. 2004, ApJ, 617, L89

De Pontieu, B., Erdélyi, R., \& James, S. P. 2004, Nature, 430, 536

Deubner, F. L., \& Fleck, B. 1989, A\&A, 213, 423

Deubner, F. L., \& Fleck, B. 1990, A\&A, 228, 506

Doyle, J. G., van den Oord, G. H. J., O'Shea, E., et al. 1999, A\&A, 347, 335

Fossum, A., \& Carlsson, M. 2006, ApJ, 646, 579

Hindman, B. W., \& Brown, T. M. 1998, ApJ, 504, 1029

Judge, P. G., Tarbell, T. D., \& Wilhelm, K. 2001, ApJ, 554, 424

Jefferies, S. M., McIntosh, S. W., Armstrong, J. D., et al. 2006, ApJ, 648, L151

Khomenko, E., Centeno, R., Collados, M., et al. 2008, ApJ, 676, L85

Kneer, F., \& von Uexküll, M. 1986, A\&A, 155, 178

Krijger, J. M., Rutten, R. J., Lites, B. W. et al. 2001, A\&A, 379, 1052

Leenarts, J., Rutten, R. J., Sütterlin, P., Carlsson, M., \& Uitenbroek H. 2006, A\&A, 449, 1209

Lites, B. W., Rutten, R. J., \& Kalkofen, W. 1993 ApJ, 414, 345L

McIntosh, S. W., \& Judge, P. G. 2001, ApJ, 561, 420

McIntosh, S. W., Fleck, B., \& Judge, P. G. 2003, A\&A, 405, 769

Moretti, P. F., Jefferies, S. M., Armstrong, J. D., et al. 2007, A\&A, 471, 961

Muglach, K. 2003, A\&A, 401, 685

Muglach, K., Hofmann, A., \& Staude, J. 2005, A\&A, 437, 1055

Reardon, K. P., Uitenbroek, H., \& Cauzzi, G. 2009, A\&A, 500, 1239

Roberts, B. 1983, Solar Phys., 87, 77

Rosenthal, C. S., Bogdan, T. J., Carlsson, M., et al. 2002, ApJ, 564, 508

Rutten, R. J. 1999, in 3rd Advances in Solar Physics Euroconference: Magnetic

Fields and Oscillations, ed. B. Schmieder, A. Hofmann, \& J. Staude, ASP Conf. Ser., 184, 181

Rutten, R. J., \& Krijger, J. M. 2003, A\&A, 407, 735

Rutten, R. J., Hammerschlag, R. H., Bettonvil, F. C. M., et al. 2004, A\&A, 413, 1183

Rutten, R. J., van Veelen, B., \& Sütterlin, P. 2008, Solar Phys., 251, 533

Suematsu, Y. 1990, Lecture Notes in Physics, 367, 211

Thomas, J. H., \& Stanchfield, D. C. H. 2000, ApJ, 537, 1086

Torrence, C., \& Compo, G. P. 1998, Bull. Amer. Meteor. Soc., 79, 61

Tsiropoula, G. 2000, New Astr., 5, 1

Tsiropoula, G., \& Tziotziou, K. 2004, A\&A, 424, 279

Tsiropoula, G., Alissandrakis, C. E., \& Schmieder, B. 1993, A\&A, 271, 574

Tsiropoula, G., Tziotziou, K., Schwartz, P., et al. 2009, A\&A, 493, 217

Tziotziou, K., Tsiropoula, G., \& Mein, P. 2003, A\&A, 402, 361

Tziotziou, K., Tsiropoula, G., \& Mein, P. 2004, A\&A, 423, 1133

Vecchio, A., Cauzzi, G., Reardon, K. P., et al. 2007, A\&A, 461, L1

Vernazza, J. E., Avrett, E. H., \& Loeser, R. 1981, ApJS, 45, 635

von Uexküll, M., Kneer, F., Malherbe, J. M., et al. 1989, A\&A, 208, 290 